\title{
Intelligent Reflecting Surfaces Enabled Cognitive Internet of Things Based on Practical Pathloss Model
}

\author{
Zheng Chu, Pei Xiao, De Mi, Hongzhi Chen, and Wanming Hao
}

\begin{abstract}
In this paper, we aim to unlock the potential of intelligent reflecting surfaces (IRSs) in cognitive internet of things (IoT). Considering that the secondary IoT devices send messages to the secondary access point (SAP) by sharing the spectrum with the primary network, the interference is introduced by the IoT devices to the primary access point (PAP) which profits from the IoT devices by pricing the interference power charged by them. A practical path loss model is adopted such that the IRSs deployed between the IoT devices and SAP serve as diffuse scatterers, but each reflected signal can be aligned with its own desired direction. Moreover, two transmission policies of the secondary network are investigated without/with a successive interference cancellation (SIC) technique. The signal-to-interference plus noise ratio (SINR) balancing is considered to overcome the near-far effect of the IoT devices so as to allocate the resource fairly among them. We propose a Stackelberg game strategy to characterize the interaction between primary and secondary networks. For the proposed game, the Stackelberg equilibrium is analytically derived to optimally obtain the closed-form solution of the power allocation and interference pricing. Numerical results are demonstrated to validate the performance of the theoretical derivations.
\end{abstract}

Index Terms-Dedicated intelligent reflecting surfaces, internet of things, practical path loss, cognitive network, Stackelberg game.

\section{INTRODUCTION}

In the emerging fifth-generation $(5 \mathrm{G})$ wireless communications, mobile traffic volume is expected to increase by a thousand-fold over the next decade. The traffic explosion will be driven by the massive increase in the number of internet of thing (IoT) devices [1]. In a generic IoT system, a number of sensor nodes link with an access point (AP) to form a wireless sensor network (WSN), which has been widely considered in variety of practical scenarios, i.e., external environment monitoring, event detection for emergency services, wireless surveillance for public safety, healthcare diagnosis, etc. [2]. In addition, cognitive radio network $(\mathrm{CRN})$ has been considered as an emerging technology which unlocks the potential to overcome the spectrum shortage. The main principle of cognitive radio for improving spectral efficiency and flexibility is

This work was supported by the U.K. Engineering and Physical Sciences Research Council under Grants EP/P008402/2 and EP/ R001588/1.

Z. Chu, P. Xiao, D. Mi, and $\mathrm{H}$. Chen are working with the Institute for Communication Systems, University of Surrey, Guildford GU2 7XH, UK. (Email: andrew.chuzheng7@gmail.com, p.xiao@surrey.ac.uk, d.mi@surrey.ac.uk, hongzhi.chen@surrey.ac.uk)

W. Hao is working with the School of Information Engineering, Zhengzhou University, Zhengzhou 450001, China. (e-mail:wmhao@ hotmail.com). that secondary users are able to opportunistically access radio resources owned by the primary network [3].

In recent years, there have been various existing works focusing on the improvement of quality of service (QoS) in $5 \mathrm{G}$ networks and beyond, it has been evolved from humancentric to machine-centric boosted by variety of requirements, e.g., ultra-high spectral efficiency and throughput. These works mainly focused on the advanced techniques to improve the spectral efficiency and throughput, e.g., massive multipleinput multiple-output (massive MIMO) with millimetre wave (mmWave), relaying, and ultra-dense networks (UDNs) [4][6]. In these advanced techniques, however, more energy consumption and hardware cost are introduced due to the use of a large number of radio frequency (RF) chains in high frequency band. Large intelligent surface brings more transmitted data and emission of radio wave [7], and evolves a new communication environment, i.e., smart radio environment. It provides a massive and seamless connectivity plus a capability of data transmission and information processing via recycling the existing radio waves instead of generating new ones [7].

As an indispensable part of smart radio environments, intelligent reflecting surface (IRS) unlocks the novel communication paradigm to improve spectral efficiency and throughput performance for the future sixth generation (6G) wireless communication networks [8]. The IRS refers to a massive reconfigurable reflecting elements that are managed by a software-oriented IRS controller [7]. These reflecting elements can efficiently reflect the intended signal without a dedicated RF processing, en/de-coding, or re-transmission since they typically embrace small, low-cost, and low-energy consumption features [9], [10]. Also, the reflected paths of the intended signal can be aligned with the direct link to maximize the signal strength by intelligently varying the phase shift of the reflecting elements [9]. Comparing with the traditional active antenna arrays, the IRS acts as a passive reflecting antenna array to improve achievable spectral efficiency and throughput in a cost-effective and energy-effective fashion and constitutes an energy self-sustainable solution.

\section{A. State of the Art}

The IRS enabled wireless networks have been drawn increasing attention in academia [9]-[13]. In [9], [10], a multiantenna downlink system was investigated under the assistance of the IRS, which is deployed to passively receives and reflects the desired signal broadcast by an access point (AP), so as 
to align with the direct link between the AP and users via intelligently varying the phase shift of each reflecting element. The results in [9], [10] show that the application of the IRS can benefit from energy saving with same spectral efficiency requirements in comparison to the the conventional multiantenna downlink system. The performance of the wireless security can also be improved by deploying the IRS [11], [12]. These works investigated the IRS enabled multi-antenna secure system, where the network power efficiency and the achievable secrecy performance are enhanced via alternately designing the secure transmit and reflecting beamforming, respectively. In [13], the IRS is invoked to enable downlink multi-cell multiple-input multiple-output (MIMO), where the spectral efficiency of the cell-edge users can be improved by mitigating the inter-cell interference. The maximization of the weighted sum rate (WSR) is formulated to jointly designing the active precoding matrices at the base stations (BSs) and the phase shifts at the IRS, subject to the power constraint of each BS and unit modulus constraint. The IRS reflects not only the wireless information transfer (WIT) service to improve the spectral efficiency, but also the wireless energy transfer (WET) service to enhance the energy efficiency for the energy user. In [14]-[16], the IRS enabled simultaneous wireless information and power transfer (SWIPT) has been studied, where the desired signal broadcast by the BS is passively reflected by the IRS in terms of information and energy simultaneously. The work in [14] discussed an emerging IRS aided SWIPT system, which enjoys a significant improvement of the WPT efficiency and characterizes a Rate-Energy tradeoff via maximizing weighted harvested energy of energy users with the individual SINR constraint for information users. A further discussion has been extended to the case with multiple IRSs [15], which are deployed for the whole information and energy transfer from a AP to information users as well as energy users, respectively. The total power consumption is minimized to guarantee the QoS constraints of all users, which highlights the energy efficiency performance of the IRSs enabled SWIPT system. An IRS assisted MIMO SWIPT system has been investigated to maximize the weighted sum rate (WSR) of the information users, satisfying the energy harvesting requirement of the energy users [16]. Alternating optimization algorithm is proposed by taking into consideration the classic block coordinate descent (BCD) algorithm, which decomposes the formulated problem into several subproblems, and alternately optimizes the transmit precoding and phase shift matrices.

Apart from the downlink scenarios, the IRS assisted multiple access channel (MAC) has also drawn increasing research attention, such as the IRS enabled MAC [17], and the IRS assisted wireless powered communication network (WPCN) user cooperation (UC) system [18]. Capacity region and deployment mechanism were investigated in the IRS enabled two-user MAC with the help of distributed and centralized IRSs [17]. The trade-off of the achievable rate between two users has been analyzed, which demonstrates that the centralized IRS deployment outperforms the distributed counterpart under the practical channel configuration. Also, the two-user cooperation in the WPCN has been studied with assistance of an IRS [18]. Particularly, one user is located nearer to the AP (i.e., central user), and the other lies farther away the AP (i.e., edge user). The AP provides wireless energy service to both users, and the central user acts as a relay to forward the information from the edge user to the AP. Additionally, the use of the IRS aims to enhance the energy efficiency in the WET phase, as well as the spectral efficiency in the WIT phase. Joint optimization of transmission time, power allocations of two users, and the phase shifts of energy/information reflection are designed to maximize the common throughput performance. Here is worth noting that mobile edge computing (MEC) is a promising technique to reduce latency in wireless transmission, where the wireless devices (WDs) located at the cell edge typically have a relatively low offloading rate.The IRS assisted MEC system was proposed to help the WDs in reducing latency as well as improving the computational offloading [19], [20], where the WDs offload a portion of their computational tasks to the AP equipped with a MEC server under the assistance of an IRS [19]. The processing latency is minimized to guarantee the constraints of the edge computing capability, which alternately designs the computing and communication configurations. In [20], the IRS assisted WPCN $\mathrm{MEC}$ in orthogonal frequency-division multiplexing (OFDM) configurations has been exploited. The network energy consumption is minimized to exploit the power allocations of the WET and offloading phases, the local computing frequencies of WDs, both binary sub-carrier allocation as well as the phase shift matrices of WET and offloading phases. These works mainly focused on the conventional phase shifts of the IRS elements, where the phase shift is designed in terms of vector or matrix optimization, or derived in terms of closed-form expression, which, however, does not provide any practical insights into the engineering system design. This motivates the work of this paper.

In this paper, we consider a practical phase shift deign which combines the practical path-loss model design. To be specific, a set of IRSs enabled cognitive radio (CR) IoT system is considered, where these IRSs assist the secondary IoT network to improve the spectral efficiency by sharing the spectrum with primary network. Meanwhile, relying on a far-field path-loss model, each element of the IRS individually plays diffuse scatterers which jointly compiles the signal from the IoT device in an intended direction with a certain beamwidth to gain the maximum signal strength. The contributions of this paper are summarized as follows:

1) We model each IRS as a rectangular plate, and investigate a practical path loss model to guarantee that each IRS aims to diffuse scatterers but the reflected signal can be aligned in the desired direction. Also, the incident and reflection angles of each element are characterized, where the IRS can be deployed in a way to gain the maximum signal strength via generating a desired observation angle instead of the reflected angle. Also, the amplitude of the path-loss model is characterized to obtain the maximum reflection gains for each element at the IRS.

2) Next, We model and investigate a set of IRSs assisted 
CR IoT system. Particularly, multiple secondary IoT devices access to the secondary AP (SAP) via the code division multiple access (CDMA), each can be served by an IRS to improve its own uplink achievable rate. Interference is introduced by each IoT device due to spectrum sharing with the primary network.

3) We generalize our model where two heterogeneous networks belong to different network operators, such that the IoT devices introduce interference pricing to the PAP. Additionally, SINR balancing condition is investigated to overcome the near-far effect of the IoT devices leading to fair resource allocation among devices. Two transmission policies are exploited with/without successive interference cancellation (SIC).

4) To shed more light on our model, we characterize the interaction between both heterogeneous networks as a Stackelberg game with the SINR balancing requirements for all IoT devices. The PAP is modelled as the leader which coordinates the interference price for each IoT device to maximize its sum utility. While the IoT device is modelled as the follower to determine the optimal power allocation, defined as the difference between sum rate achieved at the PAP and the sum cost of the payment to the PAP. For the formulated game, a Stakelberg equilibrium is derived where both leader and follower games come to an agreement on the interference pricing and power allocation at the PAP and the IoT devices.

The remainder of this paper is organized as follows. Section II describes system model. Section III investigates the game theory based formulation for the IRS assisted cognitive IoT system. In Section IV numerical results are presented to validate the theoretical results of the formulated game theory. Finally, we conclude this paper in Section V.

\section{SySTEM MODEL}

In this section, we consider a CRN as shown in Fig. 11 where $M$ cognitive users (i.e., IoT devices) transmit to the SAP with the CDMA technology via sharing the spectrum with the PAP, each of those devices is deployed with one IRS to improve the reception at the SAP. Meanwhile, the cognitive IoT devices introduce interference to the PAP. In the following, we describe the path loss model of each IRS, as well as the system model.

\section{A. Pathloss Model}

In this subsection, we exploit a novel practical path loss model for the IRS [21]. Specifically, we extract one of multiple IRSs from Fig. 1, which is considered as a rectangular surface with the size of $\alpha \times \beta$ as shown in Fig. 2, where $\alpha$ and $\beta$ are related with the wavelength $\lambda$. An IoT device radiates a linearly polarized electromagnetic wave to the surface with the incident angle $\varphi_{I} \in[0,2 \pi]$ and then the surface will reflect the desired signal with a reflecting angle $\varphi_{S} \in[0,2 \pi]$. According to the Snells law, it is expected that the observation angle $\varphi_{S}=\varphi_{I}$ to maximize the reflecting magnitude, which is the specular direction [21]. However, it is not always possible and sometimes undesirable for an IRS to reflect signals in the specular direction, due to the fact that the IRS may adjust the phases of its reflecting elements with high resolution such that a main passive reflecting beam can be generated with the desired observation angle $\varphi_{R}$. Also, the IRS will not produce any negative impact on the reflecting magnitude and beamwidth in comparison with the ideal case of a passive metallic surface [21]. By intelligently adjusting the impedance of each element, an appropriate phase can be obtained to satisfy the generalized Snells law. Thus, the IRS is employed to reflect a signal with maximum amplitude towards $\varphi_{R}$ instead of $\varphi_{I}$. Thus, path loss model of the reflecting path can be written as [21]

$$
\begin{aligned}
L_{\mathrm{IRS}}= & \frac{G_{T} G_{R}(\alpha \beta)^{2}}{\left(4 \pi d_{1} d_{2}\right)^{2}} \cos ^{2}\left(\varphi_{I}\right) * \\
& \left(\frac{\sin \left(\frac{\pi \beta}{\lambda}\left(\sin \left(\varphi_{S}\right)-\sin \left(\varphi_{R}\right)\right)\right)}{\frac{\pi \beta}{\lambda}\left(\sin \left(\varphi_{S}\right)-\sin \left(\varphi_{R}\right)\right)}\right)^{2},
\end{aligned}
$$

where $\varphi_{S} \in[0, \pi]$. In order to gain more insights into the path loss model of the reflecting path, we plot $L_{\mathrm{IRS}}$ in Fig. 3 to show the path loss amplitude versus the observation angle $\varphi_{S} \in[0, \pi]$ for different sizes of the IRS with $\varphi_{R}=60^{\circ}$. It can be observed from the figure that the maximum path loss gain is obtained when $\varphi_{S}=\varphi_{R}$ or $\pi-\varphi_{R}$. Also, the main beamwidth becomes narrower with a larger IRSs surface size so as to enhance the reflecting amplitude of the IRS. When $\alpha$ and $\beta$ satisfy $\alpha, \beta \leq \frac{\lambda}{2}$, the IRS almost acts as a diffuse scatterer. Additionally, when $\varphi_{S}=\varphi_{R}$ or $\pi-\varphi_{R}$ holds, the path loss maps to the maximum reflection gain, which can be simplified as

$$
L_{\mathrm{IRS}}=\frac{G_{T} G_{R}(\alpha \beta)^{2}}{\left(4 \pi d_{1} d_{2}\right)^{2}} \cos ^{2}\left(\varphi_{I}\right),
$$

From (2), the path loss gain $L_{\mathrm{IRS}}$ depends on the IRS's area $\alpha \beta$ and incident angle $\varphi_{I}$ from the transmitter to the IRS. When the IRS is composed by large amount of reflecting elements $N=N_{\alpha} \times N_{\beta}$, where $N_{\alpha}$ and $N_{\beta}$ are the element numbers of $\alpha$ and $\beta$, respectively. The size of each element is $\frac{\alpha \beta}{N_{\alpha} N_{\beta}}$. Thus, we can express the path loss between the transmitter and receiver through the $n$-th surface element as [21]

$$
L_{\mathrm{IRS}}^{\prime}=\frac{G_{T} G_{R}(\alpha \beta)^{2}}{\left(4 \pi N_{\alpha} N_{\beta} d_{1} d_{2}\right)^{2}} \cos ^{2}\left(\varphi_{I}\right)
$$

Also, the path loss between the transmitter and receiver at the whole IRS can be written as

$$
L_{\mathrm{IRS}}=N^{2} \frac{G_{T} G_{R}(\alpha \beta)^{2}}{\left(4 \pi N_{\alpha} N_{\beta} d_{1} d_{2}\right)^{2}} \cos ^{2}\left(\varphi_{I}\right)=N^{2} L_{\mathrm{IRS}}^{\prime}
$$

\section{B. System Description}

In this subsection, we describe the system model as shown in Fig. 1. Specifically, we assume that the channels between the $m$-th IoT and SAP, the $m$-th IoT device and PAP, as well as the $m$-th IoT device and the SAP via the $m$-th IRS are line-of-sight (LoS), denoted by $\sqrt{L_{d, m}} h_{d, m}, \sqrt{L_{p, m}}$, and $\sqrt{L_{\mathrm{IRS}_{m}}} \mathbf{h}_{m}^{T} \boldsymbol{\Theta}_{m} \mathbf{g}_{m}$, respectively, where $L_{d, m}=\frac{G_{T} G_{R} \lambda^{2}}{\left(4 \pi d_{s, m}\right)^{2}}$, $L_{p, m}=\frac{G_{T} G_{R} \lambda^{2}}{\left(4 \pi d_{p, m}\right)^{2}}, \quad L_{\mathrm{IRS}, m}^{\prime}=\frac{G_{T} G_{R}(\alpha \beta)^{2}}{\left(4 \pi d_{m} d_{S A P, m}\right)^{2}} \cos ^{2}\left(\varphi_{I, m}\right)$. Also, $d_{s, m}, d_{p, m}, d_{m}, d_{S A P, m}$ denote the distance between the $m$-th IoT device and the SAP, the $m$-th IoT device and the PAP, the $m$-th IoT device and the $m$-th IRS, as well as the $m$-th IRS and the SAP, respectively. In this paper, we 


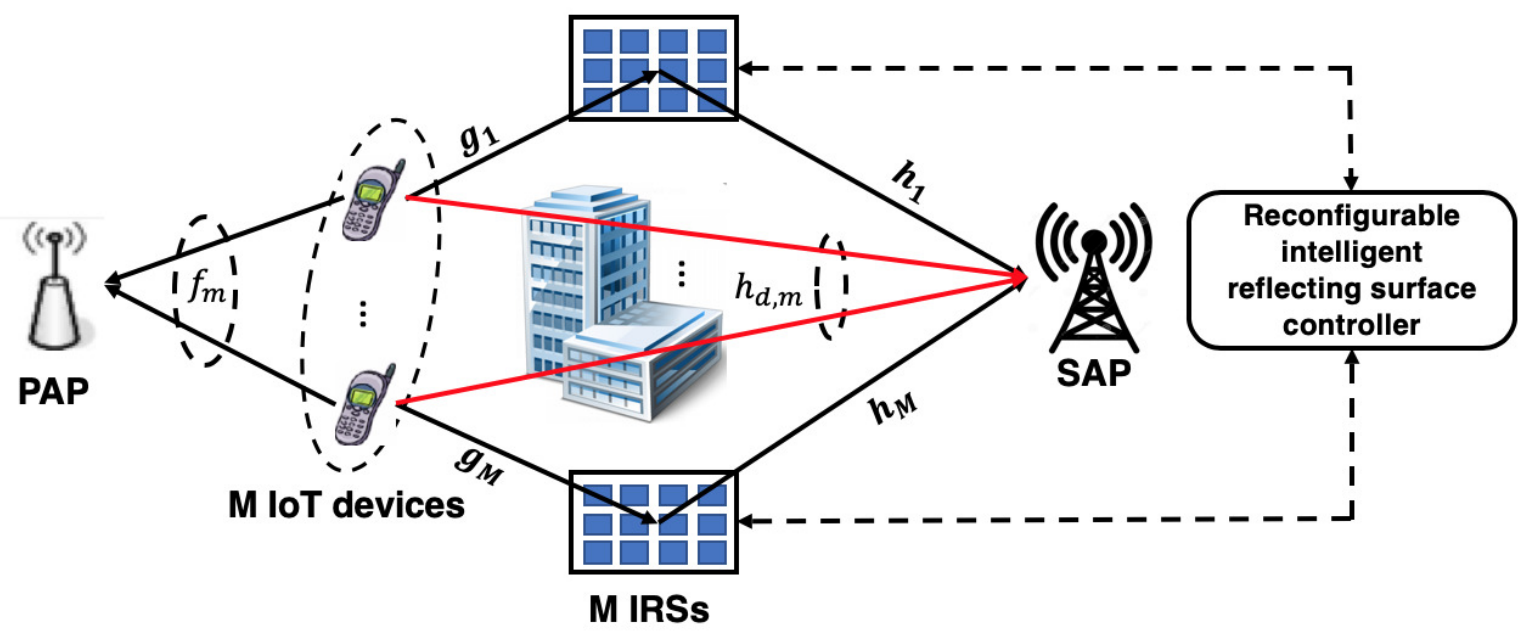

Fig. 1: System model.

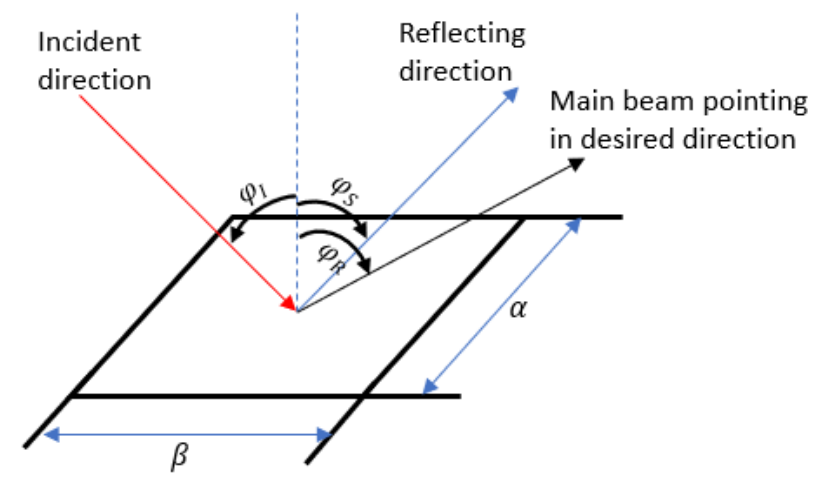

Fig. 2: Reflecting surface model.

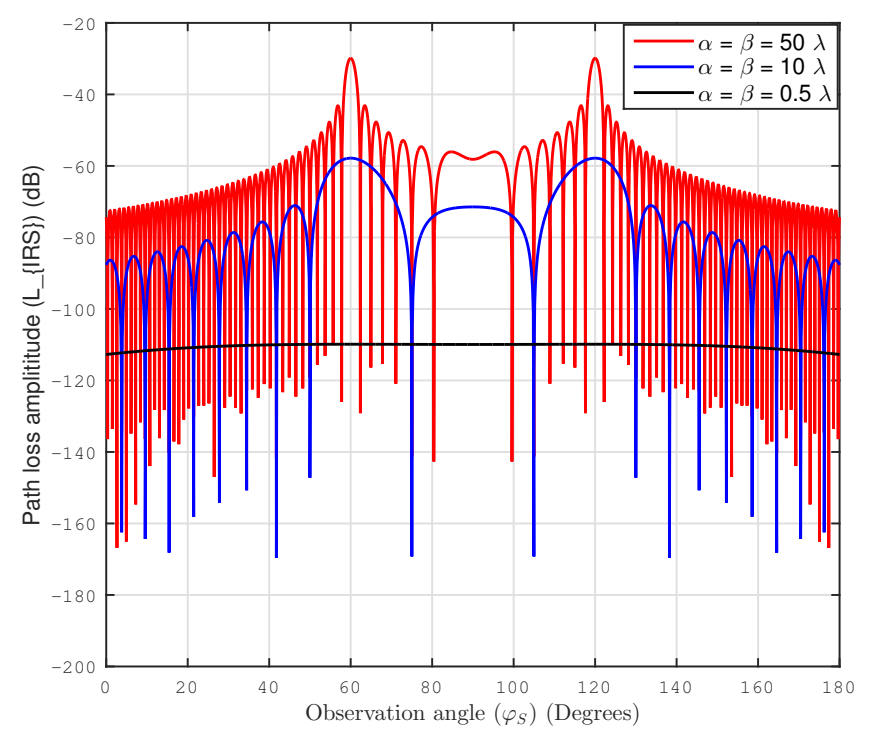

Fig. 3: Path loss amplitude versus observation angle $\varphi_{S}$ assume perfect knowledge of the channel state information (CSI) due to the fact that we are interested in achieving an upper bound of the sum utility performance. In practice, the CSI can be obtained through different methods such as the CSI feedback method [22] or even the local oscillator power leakage from the RF frontend at the receiver [23]. In addition, the IRS controller is generally fixed to coordinate its working modes, i.e., reception for channel estimation as well as reflection modes for information reflection, respectively [9], via intelligently controlling its phase shifts. In addition, $\varphi_{I, m}$ is the incident angle from the $m$-th IoT device to the $m$ th IRS. Moreover, $\mathbf{h}_{m}=\left[\exp \left(j \phi_{m, 1}\right), \ldots, \exp \left(j \phi_{m, N}\right)\right]^{T}$ and $\mathbf{g}_{m}=\left[\exp \left(j \theta_{m, 1}\right), \ldots, \exp \left(j \theta_{m, N}\right)\right]^{T}$ represent the normalized LoS channels between the $m$-th IRS and the SAP as well as the $m$-th IoT device and the $m$-th IRS, respectively. $\phi_{m, n}$ and $\theta_{m, n}(n \in[1, N])$ are the phase of the associated channel. While $\boldsymbol{\Theta}_{m}=\operatorname{Diag}\left[\exp \left(j \alpha_{m, 1}\right), \ldots, \exp \left(j \alpha_{m, N}\right)\right]$ is phase shift matrix of the $k$-th IRS, where $\alpha_{m, n}(n \in[1, N])$ denotes the reflecting coefficient at the $n$ element of the $m$-th IRS. Thus, the received signal at the SAP is written as

$$
\begin{aligned}
y=\sum_{m=1}^{M} \sqrt{P_{m}}\left[\sqrt{L_{\mathrm{IRS}_{m}}^{\prime}} \mathbf{h}_{k}^{T} \boldsymbol{\Theta}_{k} \mathbf{g}_{m}+\sqrt{L_{d, m}} h_{d, m}\right] x_{m}+z \\
=\sum_{m=1}^{M} \sqrt{P_{m}}\left[\sqrt{L_{\mathrm{IRS}, m}^{\prime}} \sum_{i=1}^{N} \exp \left[j\left(\theta_{m, i}+\alpha_{m, i}+\phi_{m, i}\right)\right]\right. \\
\left.+\sqrt{L_{d, m}} \exp \left(j \beta_{m}\right)\right] x_{m}+z \\
=\sqrt{P_{m}}\left[\sqrt{L_{\mathrm{IRS}, m}^{\prime}} \sum_{i=1}^{N} \exp \left[j\left(\theta_{m, i}+\alpha_{m, i}+\phi_{m, i}\right)\right]\right. \\
\left.+\sqrt{L_{d, m}} \exp \left(j \beta_{m}\right)\right] x_{m} \\
+\sum_{n \neq m} \sqrt{P_{n}}\left[\sqrt{L_{\mathrm{IRS}, n}^{\prime}} \sum_{i=1}^{N} \exp \left[j\left(\theta_{n, i}+\alpha_{n, i}+\phi_{n, i}\right)\right]\right. \\
\left.+\sqrt{L_{d, n}} \exp \left(j \beta_{n}\right)\right] x_{n}+z
\end{aligned}
$$


where $P_{m}$ denotes the transmit power at the $m$-th IoT device, and $z$ is the the additive white Gaussian noise (AWGN) with zero mean and variance $\sigma^{2}$. In order to maximize the received signal power at the SAP, the phase of reflecting signal needs to be aligned with that of the direct signal. Thus, we select $\beta_{m}=\theta_{m, i}+\alpha_{m, i}+\phi_{m, i}$ such that the received signal in (5) is rewritten as

$$
\begin{aligned}
y= & \sqrt{P_{m}}\left[N \sqrt{L_{\mathrm{IRS}, m}^{\prime}}+\sqrt{L_{d, m}}\right] \exp \left(j \beta_{m}\right) x_{m} \\
& +\sum_{n \neq m} \sqrt{P_{n}}\left[N \sqrt{L_{\mathrm{IRS}, n}^{\prime}}+\sqrt{L_{d, n}}\right] \exp \left(j \beta_{n}\right) x_{n}+z, \\
= & \sqrt{P_{m}}\left[\sqrt{L_{\mathrm{IRS}, m}}+\sqrt{L_{d, m}}\right] \exp \left(j \beta_{m}\right) x_{m} \\
& +\sum_{n \neq m} \sqrt{P_{n}}\left[\sqrt{L_{\mathrm{IRS}, n}}+\sqrt{L_{d, n}}\right] \exp \left(j \beta_{n}\right) x_{n}+z,
\end{aligned}
$$

To proceed, we consider the following two cases of different decoding schemes.

Without SIC: The SINR of the SAP can be written as

$$
\mathrm{SINR}_{m}^{\mathrm{WOSIC}}=\frac{S P_{m}\left[\sqrt{L_{\mathrm{IRS}, m}}+\sqrt{L_{d, m}}\right]^{2}}{\sum_{n \neq m} P_{n}\left[\sqrt{L_{\mathrm{IRS}, n}}+\sqrt{L_{d, n}}\right]^{2}+\sigma^{2}}
$$

where $S=C / D>1$ is the spreading gain of the CDMA system, $C$ is the chip rate, $D$ is the data rate [24]. The associate achievable rate is given by

$$
R_{m}=\log _{2}\left(1+\operatorname{SINR}_{m}^{\mathrm{WOSIC}}\right),
$$

where $\sqrt{L_{\mathrm{IRS}, m}}=N \sqrt{L_{\mathrm{IRS}, m}^{\prime}}$.

With SIC: We index the descending order of IoT devices from 1 to $M$ in terms of decoding. With the SIC technique, the strong devices will cancel the interference from the weaker counterparts. The SIC technique is typically implemented in a non-orthogonal multiple access (NOMA) system. Thus, the SINR can be derived as

$$
\mathrm{SINR}_{m}^{\mathrm{SIC}}=\frac{S P_{m}\left[\sqrt{L_{\mathrm{IRS}, m}}+\sqrt{L_{d, m}}\right]^{2}}{\sum_{n>m} P_{n}\left[\sqrt{L_{\mathrm{IRS}, n}}+\sqrt{L_{d, n}}\right]^{2}+\sigma^{2}},
$$

and its associate achievable rate is given by

$$
R_{m}=\log _{2}\left(1+\operatorname{SINR}_{m}^{\mathrm{SIC}}\right) \text {. }
$$

Also, the cognitive IoT devices introduce the interference to the PAP, which can be expressed as

$$
I_{m}=P_{m} L_{p, m}, \forall m \in[1, M] \text {. }
$$

\section{FAIRnESS AWARE AND IRS Assisted Cognitive INTERNET OF THINGS}

\section{A. Game Theory Formulation}

In this subsection, we exploit the strategy between the cognitive network and the primary network, and model the strategy between the PAP and cognitive IoT devices as a Stackelberg game.

Leader: The PAP is modelled as the leader in the Stackelberg game that decides a price for each IoT device to maximize its sum utility under interference power constraint (IPC). To proceed, we write the sum utility of the PAP as

$$
U_{L}(\boldsymbol{\mu}, \mathbf{p})=\sum_{m=1}^{M} \mu_{m} P_{m} L_{p, m}
$$

where $\mathbf{p}=\left[P_{1}, \ldots, P_{M}\right]$, and $\mu_{m}$ is the price for unit interference power decided for the $m$-th IoT device. Also, the leader game can be formulated as

$$
\begin{aligned}
\max _{\boldsymbol{\mu}} & U_{L}(\boldsymbol{\mu}, \mathbf{p}), \\
\text { s.t. } & \boldsymbol{\mu} \succeq \mathbf{0}, \mathbf{p} \succeq \mathbf{0}, \operatorname{SINR}_{m}(\mathbf{p})=\operatorname{SINR}_{n}(\mathbf{p}), n \neq m, \\
& \sum_{m=1}^{M} P_{m} L_{p, m} \leq I_{t h},
\end{aligned}
$$

where $I_{t h}$ is the interference power budget at the PAP, and $\mathrm{SINR}_{m}$ denotes the SINR of the scheme with/without case.

Follower: The IoT devices are the followers that determine the optimal power allocation to maximize their sum utility which is defined as the difference between the revenue of the sum rate achieved at the SAP and the sum cost of the payment to the PAP. The sum utility of the SAP can be written as

$$
U_{F}(\boldsymbol{\mu}, \mathbf{p})=\sum_{m=1}^{M} \lambda_{m} \log _{2}\left(1+\operatorname{SINR}_{m}(\mathbf{p})\right)-\sum_{m=1}^{M} \mu_{m} P_{m} L_{p, m}
$$

Also, the follower game is written as

$$
\begin{aligned}
\max _{\mathbf{p}} & U_{F}(\boldsymbol{\mu}, \mathbf{p}) \\
\text { s.t. } & \mathbf{p} \succeq \mathbf{0}, \\
& \operatorname{SINR}_{m}(\mathbf{p})=\operatorname{SINR}_{n}(\mathbf{p}), n \neq m .
\end{aligned}
$$

Note that constraint $(15 \mathrm{~b})$ is the SINR balancing condition to overcome the near-far effect of IoT devices so as to allocate the resource fairly among these IoT devices. Problem (13) and problem (15) form a Stackelberg game, which aims to obtain the maximum revenues of the leader and follower games. This formulated game can be solved via taking into consideration Stackelberg equilibrium.

To solve the formulated Stackelberg game in (13) and (15), we adapt the well-known Stackelberg equilibrium to derive the solution of the formulated game, which is defined as the following definition

Definition 1: Let $\boldsymbol{\mu}^{*}$ and $\mathbf{p}^{*}$ denote the solutions of problems (13) and (15). The set $\left(\boldsymbol{\mu}^{*}, \mathbf{p}^{*}\right)$ is a Stackelberg equilibrium of the formulated game ensuring that the following conditions are guaranteed

$$
U_{L}\left(\boldsymbol{\mu}^{*}, \mathbf{p}^{*}\right) \geq U_{L}\left(\boldsymbol{\mu}, \mathbf{p}^{*}\right), U_{F}\left(\boldsymbol{\mu}^{*}, \mathbf{p}^{*}\right) \geq U_{F}\left(\boldsymbol{\mu}^{*}, \mathbf{p}\right) .
$$

In the following, we will derive the Stackelberg equilibrium for the proposed game, where the optimal solutions to problem (13) and (15) are derived in closed-form expressions to maximize their own sum utility.

\section{B. Solution for Stackelberg Game}

In this subsection, we solve problem (13) and problem 15 via the Stackelberg equilibrium shown in Definition 1 .

1) Without SIC: We first derive the Stackelberg equilibrium for the scheme without SIC. The following lemma is required.

Lemma 1: In the scheme without SIC, let $\left(P_{1}, \ldots, P_{M}\right)$ be the transmit power of the IoT devices, the cognitive network charges for the interference pricing $\mu_{m}(m=1, \ldots, M)$ for 
the $m$-th IoT device, and the term $P_{m}\left[\sqrt{L_{\mathrm{IRS}, m}}+\sqrt{L_{d, m}}\right]^{2}$ satisfies following equality:

$$
\begin{gathered}
{\left[\begin{array}{cccc}
1 & \frac{1}{S} & \cdots & \frac{1}{S} \\
\frac{1}{S} & 1 & \ddots & \frac{1}{S} \\
\vdots & \vdots & \ddots & \vdots \\
\frac{1}{S} & \frac{1}{S} & \cdots & 1
\end{array}\right]\left[\begin{array}{c}
P_{1}\left[\sqrt{L_{\mathrm{IRS}, 1}}+\sqrt{L_{d, 1}}\right]^{2} \\
P_{2}\left[\sqrt{L_{\mathrm{IRS}, 2}}+\sqrt{L_{d, 2}}\right]^{2} \\
\vdots \\
P_{M}\left[\sqrt{L_{\mathrm{IRS}, M}}+\sqrt{L_{d, M}}\right]^{2}
\end{array}\right]} \\
=\left[\begin{array}{c}
\frac{\lambda_{1}\left[\sqrt{L_{\mathrm{IRS}, 1}}+\sqrt{L_{d, 1}}\right]^{2}}{\mu_{1} L_{p, 1}}-\frac{\sigma^{2}}{S} \\
\frac{\lambda_{2}\left[\sqrt{L_{\mathrm{IRS}, 2}}+\sqrt{L_{d, 2}}\right]^{2}}{\mu_{2} L_{p, 2}}-\frac{\sigma^{2}}{S} \\
\vdots \\
\frac{\lambda_{M}\left[\sqrt{L_{\mathrm{IRS}, M}}+\sqrt{L_{d, M}}\right]^{2}}{\mu_{M} L_{p, M}}-\frac{\sigma^{2}}{S}
\end{array}\right]
\end{gathered}
$$

Proof: See Appendix A

By exploiting Lemma 1, we multiple both sides of (39) in Appendix A by $P_{m}$, which is given by (17) on the top of next page. Note that the utility of the SAP obtained from the $m$-th IoT device can be expressed as the left hand side of (17) such that $P_{m}(m=1, \ldots, M)$ satisfies (16) for a given price $\mu_{m}$ $(m=1, \ldots, M)$. We substitute (17) into the leader game in (13), which is given by (18) on the top of the next page. By exploiting the fairness constraint (18a), the following lemma is required:

Lemma 2: For every IoT device, the SINR balancing constraint 18a holds, $P_{m}\left[\sqrt{L_{\mathrm{IRS}, m}}+\sqrt{L_{d, m}}\right]^{2}=$ $P_{n}\left[\sqrt{L_{\mathrm{IRS}, n}}+{\sqrt{L_{d, n}}}^{2}\right.$ is hold.

Proof: See Appendix B

Lemma 2 evaluates that the receive power from each IoT device to the SAP is identical under the SINR balancing condition constraint $\operatorname{SINR}_{m}(\mathbf{p})=\operatorname{SINR}_{n}(\mathbf{p})$. To proceed, we define $X=P_{m}\left[\sqrt{L_{\mathrm{IRS}, m}}+\sqrt{L_{d, m}}\right]^{2}$, and reformulate the leader game 13 as

$$
\begin{aligned}
& \max _{X} \frac{S X}{(M-1+S) X+\sigma^{2}} \sum_{m=1}^{M} \lambda_{m} \\
& \text { s.t. } X \sum_{m=1}^{M} \frac{L_{p, m}}{\left[\sqrt{L_{\mathrm{IRS}, m}}+\sqrt{L_{d, m}}\right]^{2}} \leq I_{t h} .
\end{aligned}
$$

It can be easily verified that the objective function (19a) is monotonically increasing with respect to $X$ via take into consideration its first-order derivative greater than zero. In order to maximize the objective function (19a), we need to consider the equality of the constraint (19b). Thus, the optimal solution of $X$ can be written in closed-form expression as

$$
X^{*}=\frac{I_{t h}}{\sum_{m=1}^{M} \frac{L_{p, m}}{\left[\sqrt{L_{\mathrm{IRS}, m}}+\sqrt{L_{d, m}}\right]^{2}}} .
$$

Consequently, the optimal solution of $P_{m}$ is given by

$$
P_{m}^{*}=\frac{I_{t h}}{\left[\sqrt{L_{\mathrm{IRS}, m}}+\sqrt{L_{d, m}}\right]^{2} \sum_{m=1}^{M} \frac{L_{p, m}}{\left[\sqrt{L_{\mathrm{IRS}, m}}+\sqrt{L_{d, m}}\right]^{2}}} .
$$

According to (17), the optimal price $\mu_{m}$ can be derived in closed-form expression as

$$
\mu_{m}^{*}=\frac{\lambda_{m} S\left[\sum_{k=1}^{K} \sqrt{L_{\mathrm{IRS}, m k}}+\sqrt{L_{d, m}}\right]^{2}}{L_{p, m}\left[(M-1+S) X^{*}+\sigma^{2}\right]}
$$

2) With SIC: In this subsection, we take into consideration the derivation of Stackelberg equilibrium for the scheme with SIC. First, the following Lemma is required

Lemma 3: In the scheme with SIC, let $\left(P_{1}, \ldots, P_{M}\right)$ be the transmit power of the IoT devices, the cognitive network charges for the interference pricing $\mu_{m}(m=1, \ldots, M)$ for the $m$-th IoT device, and the term $P_{m}\left[\sqrt{L_{\mathrm{IRS}, m}}+\sqrt{L_{d, m}}\right]^{2}$ satisfies following equality:

$$
\begin{aligned}
& {\left[\begin{array}{cccc}
1 & \frac{1}{S} & \cdots & \frac{1}{S} \\
0 & 1 & \ddots & \frac{1}{S} \\
\vdots & \vdots & \ddots & \vdots \\
0 & 0 & \cdots & 1
\end{array}\right]\left[\begin{array}{c}
P_{1}\left[\sqrt{L_{\mathrm{IRS}, 1}}+\sqrt{L_{d, 1}}\right]^{2} \\
P_{2}\left[\sqrt{L_{\mathrm{IRS}, 2}}+\sqrt{L_{d, 2}}\right]^{2} \\
\vdots \\
P_{M}\left[\sqrt{L_{\mathrm{IRS}, M}}+\sqrt{L_{d, M}}\right]^{2}
\end{array}\right]} \\
& =\left[\begin{array}{c}
\frac{\lambda_{1}\left[\sqrt{L_{\mathrm{IRS}, 1}}+\sqrt{L_{d, 1}}\right]^{2}}{\mu_{1} L_{p, 1}}-\frac{\sigma^{2}}{S} \\
\frac{\lambda_{2}\left[\sqrt{L_{\mathrm{IRS}, 2}}+\sqrt{L_{d, 2}}\right]^{2}}{\mu_{2} L_{p, 2}}-\frac{\sigma^{2}}{S} \\
\vdots \\
\frac{\lambda_{M}\left[\sqrt{L_{\mathrm{IRS}, M}}+\sqrt{L_{d, M}}\right]^{2}}{\mu_{M} L_{p, M}}-\frac{\sigma^{2}}{S}
\end{array}\right] .
\end{aligned}
$$

Proof: See the proof of Lemma 1 .

By setting the first derivative of (14) to zero, we have (24) on the top of the next page. Substitute (24) into the leader game (13) with SIC, we obtain 25) on the top of the next page. In order to solve problem 25], we first tackle the SINR balancing constraint $\operatorname{SINR}_{m}(\mathbf{p})=\operatorname{SINR}_{n}(\mathbf{p})$. More specifically, denote $\gamma_{m}=\frac{S P_{m}\left[\sqrt{L_{\mathrm{IRS}, m}}+\sqrt{L_{d, 1}}\right]^{2}}{\sum_{n>m} P_{n}\left[\sqrt{L_{\mathrm{IRS}, n}}+\sqrt{L_{d, n}}\right]^{2}+\sigma^{2}}$, the following equalities hold

$$
\begin{aligned}
& \frac{S P_{1}\left[\sqrt{L_{\mathrm{IRS}, 1}}+\sqrt{L_{d, 1}}\right]^{2}}{\sum_{n>1} P_{n}\left[\sqrt{L_{\mathrm{IRS}, n}}+\sqrt{L_{d, n}}\right]^{2}+\sigma^{2}} \\
& =\ldots=\frac{S P_{M-1}\left[\sqrt{L_{\mathrm{IRS}, M-1}}+\sqrt{L_{d, M-1}}\right]^{2}}{P_{M}\left[\sqrt{L_{\mathrm{IRS}, M}}+\sqrt{L_{d, n}}\right]^{2}+\sigma^{2}} \\
& =\frac{S P_{M}\left[\sqrt{L_{\mathrm{IRS}, M}}+\sqrt{L_{d, M}}\right]^{2}}{\sigma^{2}} .
\end{aligned}
$$

By letting $\gamma_{m}=\gamma,(m=1, \ldots, M-1)$ and 26 , we further derive the following equality

$$
\begin{aligned}
& \frac{S P_{m}\left[\sqrt{L_{\mathrm{IRS}, m}}+\sqrt{L_{d, m}}\right]^{2}}{\sum_{n>m} P_{n}\left[\sqrt{L_{\mathrm{IRS}, n}}+\sqrt{L_{d, n}}\right]^{2}+\sigma^{2}} \\
& =\frac{S P_{m+1}\left[\sqrt{L_{\mathrm{IRS}, m+1}}+\sqrt{L_{d, m+1}}\right]^{2}}{\sum_{n>m+1} P_{n}\left[\sqrt{L_{\mathrm{IRS}, n}}+\sqrt{L_{d, n}}\right]^{2}+\sigma^{2}}=\gamma .
\end{aligned}
$$

By exploiting a few of mathematical manipulations, we have (28) on the top of the next page. To proceed, the received power from the $m$-th IoT device $P_{m}\left[\sqrt{L_{\mathrm{IRS}, m}}+\sqrt{L_{d, m}}\right]^{2}$ 


$$
\begin{gathered}
\frac{\lambda_{m} S P_{m}\left[\sqrt{L_{\mathrm{IRS}, m}}+\sqrt{\left.L_{d, m}\right]^{2}}\right.}{\sum_{n \neq m} P_{n}\left[\sqrt{L_{\mathrm{IRS}, n}}+\sqrt{L_{d, n}}\right]^{2}+\sigma^{2}+S P_{m}\left[\sqrt{L_{\mathrm{IRS}, m}}+\sqrt{L_{d, m}}\right]^{2}}=\mu_{m} P_{m} L_{p, m} . \\
\max _{\mathbf{p}} \sum_{m=1}^{M} \frac{\lambda_{m} S P_{m}\left[\sqrt{L_{\mathrm{IRS}, m}}+\sqrt{L_{d, m}}\right]^{2}}{\sum_{n \neq m} P_{m}\left[\sqrt{L_{\mathrm{IRS}, n}}+\sqrt{L_{d, n}}\right]^{2}+\sigma^{2}+S P_{m}\left[\sqrt{L_{\mathrm{IRS}, m}}+\sqrt{L_{d, m}}\right]^{2}} \\
\text { s.t. } \operatorname{SINR}_{m}(\mathbf{p})=\operatorname{SINR}_{n}(\mathbf{p}), n \neq m, \mathbf{p} \succeq \mathbf{0}, \\
\frac{\partial U_{F}}{\partial P_{m}}=\frac{\lambda_{m} S\left[\sqrt{L_{\mathrm{IRS}, m}}+\sqrt{L_{d, m}}\right]^{2}}{\sum_{n>m} P_{n}\left[\sqrt{L_{\mathrm{IRS}, n}}+\sqrt{L_{d, n}}\right]^{2}+\sigma^{2}+S P_{m}\left[\sqrt{L_{\mathrm{IRS}, m}}+\sqrt{L_{d, m}}\right]^{2}}-\mu_{m} L_{p, m}=0, \\
\Rightarrow \frac{\lambda_{m} S P_{m}\left[\sqrt{L_{\mathrm{IRS}, m}}+\sqrt{L_{d, m}}\right]^{2}}{\sum_{n>m} P_{n}\left[\sqrt{L_{\mathrm{IRS}, n}}+\sqrt{L_{d, n}}\right]^{2}+\sigma^{2}+S P_{m}\left[\sqrt{L_{\mathrm{IRS}, m}}+\sqrt{L_{d, m}}\right]^{2}}=\mu_{m} P_{m} L_{p, m} . \\
\max _{\mathbf{p}} \sum_{m=1}^{M} \frac{\lambda_{m} S P_{m}\left[\sqrt{L_{\mathrm{IRS}, m}}+\sqrt{L_{d, m}}\right]^{2}}{\sum_{n>m} P_{n}\left[\sqrt{L_{\mathrm{IRS}, n}}+\sqrt{L_{d, n}}\right]^{2}+\sigma^{2}+S P_{m}\left[\sqrt{L_{\mathrm{IRS}, m}}+\sqrt{L_{d, m}}\right]^{2}} \\
\text { s.t. } \sum_{m=1}^{M} P_{m} L_{p, m} \leq I_{t h}, \operatorname{SINR}{ }_{m}(\mathbf{p})=\operatorname{SINR}_{n}(\mathbf{p}), n \neq m, \mathbf{p} \succeq \mathbf{0},
\end{gathered}
$$

at the SAP can be derived as

$$
\begin{aligned}
& P_{m}\left[\sqrt{L_{\mathrm{IRS}, m}}+\sqrt{L_{d, m}}\right]^{2} \\
& =\left(1+\frac{\gamma}{S}\right) P_{m+1}\left[\sqrt{L_{\mathrm{IRS}, m+1}}+\sqrt{L_{d, m+1}}\right]^{2} \\
& =\left(1+\frac{\gamma}{S}\right)^{2} P_{m+2}\left[\sqrt{L_{\mathrm{IRS}, m+2}}+\sqrt{L_{d, m+2}}\right]^{2} \\
& \vdots \\
& =\left(1+\frac{\gamma}{S}\right)^{M-m} P_{M}\left[\sqrt{L_{\mathrm{IRS}, M}}+\sqrt{L_{d, M}}\right]^{2} \\
& =\left(1+\frac{\gamma}{S}\right)^{M-m} \frac{\gamma \sigma^{2}}{S} .
\end{aligned}
$$

Also, for $m=M$, it is easily obtained from (26)

$$
P_{M}\left[\sqrt{L_{\mathrm{IRS}, M}}+\sqrt{L_{d, M}}\right]^{2}=\frac{\gamma \sigma^{2}}{S}
$$

Thus, we combine (29) and (30) to achieve the sum received power as

$$
\begin{aligned}
& \sum_{m=1}^{M} P_{m}\left[\sqrt{L_{\mathrm{IRS}, m}}+\sqrt{L_{d, m}}\right]^{2} \\
& =\sum_{m=1}^{M}\left(1+\frac{\gamma}{S}\right)^{M-m} \frac{\gamma \sigma^{2}}{S}=\left[\left(1+\frac{\gamma}{S}\right)^{M}-1\right] \sigma^{2} .
\end{aligned}
$$

Substituting 290 and (30) into the objective function (25a), yields

$$
\sum_{m=1}^{M} \frac{\lambda_{m} S\left(1+\frac{\gamma}{S}\right)^{M-m} \frac{\gamma \sigma^{2}}{S}}{\sum_{n>m}\left(1+\frac{\gamma}{S}\right)^{M-n} \frac{\gamma \sigma^{2}}{S}+\sigma^{2}+S\left(1+\frac{\gamma}{S}\right)^{M-m} \frac{\gamma \sigma^{2}}{S}}
$$

We exploit the following identity to tackle the objective function 32

$$
\begin{array}{rl}
\sum_{n>m}\left(1+\frac{\gamma}{S}\right)^{M-n} & 1+\left(1+\frac{\gamma}{S}\right)+\left(1+\frac{\gamma}{S}\right)^{2} \\
= & +\ldots+\left(1+\frac{\gamma}{S}\right)^{M-(m+2)}+\left(1+\frac{\gamma}{S}\right)^{M-(m+1)} \\
= & \sum_{i=0}^{M-(m+1)}\left(1+\frac{\gamma}{S}\right)^{i}=\frac{\left(1+\frac{\gamma}{S}\right)^{M-m}-1}{\left(1+\frac{\gamma}{S}\right)-1} \\
= & \frac{\left(1+\frac{\gamma}{S}\right)^{M-m}-1}{\frac{\gamma}{S}} .
\end{array}
$$

By substituting (33) into (32), we have

$$
\begin{aligned}
& \sum_{m=1}^{M} \frac{\lambda_{m} S\left(1+\frac{\gamma}{S}\right)^{M-m} \frac{\gamma \sigma^{2}}{S}}{\left[\left(1+\frac{\gamma}{S}\right)^{M-m}-1\right] \sigma^{2}+\sigma^{2}+\left(1+\frac{\gamma}{S}\right)^{M-m} \frac{\gamma \sigma^{2}}{S}} \\
& \quad=\frac{\gamma}{1+\gamma} \sum_{m=1}^{M} \lambda_{m}
\end{aligned}
$$

Thus, problem 25 can be reformulated as

$$
\begin{aligned}
\max _{\gamma} & \frac{\gamma}{1+\gamma} \sum_{m=1}^{M} \lambda_{m} \\
\text { s.t. } & \sum_{m=1}^{M}\left(1+\frac{\gamma}{S}\right)^{M-m} \frac{\gamma \sigma^{2}}{S} \frac{L_{P, m}}{\left[\sqrt{L_{\mathrm{IRS}, m}}+\sqrt{L_{d, m}}\right]^{2}} \leq I_{t h} .
\end{aligned}
$$

It can be easily verified that the objective function of problem (35) is monotonically increasing with respect to $\gamma$. Thus, 


$$
\begin{aligned}
& \frac{P_{m}\left[\sqrt{L_{\mathrm{IRS}, m}}+\sqrt{L_{d, m}}\right]^{2}}{P_{m+1}\left[\sqrt{L_{\mathrm{IRS}, m+1}}+\sqrt{L_{d, m+1}}\right]^{2}}=\frac{\sum_{n>m} P_{n}\left[\sqrt{L_{\mathrm{IRS}, n}}+\sqrt{L_{d, n}}\right]^{2}+\sigma^{2}}{\sum_{n>m+1} P_{n}\left[\sqrt{L_{\mathrm{IRS}, n}}+\sqrt{L_{d, n}}\right]^{2}+\sigma^{2}} \\
& =\frac{\sum_{n>m+1} P_{n}\left[\sqrt{L_{\mathrm{IRS}, n}}+\sqrt{L_{d, n}}\right]^{2}+\sigma^{2}+P_{m}\left[\sqrt{L_{\mathrm{IRS}, m}}+\sqrt{L_{d, m}}\right]^{2}}{\sum_{n>m+1} P_{n}\left[\sqrt{L_{\mathrm{IRS}, n}}+\sqrt{L_{d, n}}\right]^{2}+\sigma^{2}}=1+\frac{\gamma}{S} .
\end{aligned}
$$

the closed-form solution to $\gamma$ can be derived via taking into consideration the constraint in 35, where the bisections search can be employed to solve the following equality

$$
\sum_{m=1}^{M}\left(1+\frac{\gamma}{S}\right)^{M-m} \frac{\gamma \sigma^{2}}{S} \frac{L_{P, m}}{\left[\sqrt{L_{\mathrm{IRS}, m}}+\sqrt{\left.L_{d, m}\right]^{2}}\right.}=I_{t h} .
$$

By numerically solving the optimal solution of $\gamma$ (denoted by $\gamma^{*}$ ), the optimal solution of transmit power $P_{m}$ can be expressed as

$$
P_{m}^{*}= \begin{cases}\frac{\left(1+\frac{\gamma^{*}}{S}\right)^{M-m} \frac{\gamma^{*} \sigma^{2}}{S}}{\left[\sqrt{L_{\mathrm{RRS}, m}}+\sqrt{L_{d, m}}\right]^{2}}, & m=1, \ldots, M-1, \\ \frac{\gamma^{*} \sigma^{2}}{S} & m=M .\end{cases}
$$

We plug (37) into [24], the optimal price $\mu_{m}^{*}$ can be derived in closed-form (38) on the top of the next page.

\section{Discussion}

In this subsection, we discuss the Stackelberg equilibrium derived in Section III-B1 and Section III-B2. Specifically, the optimal solutions of the power allocation and the interference pricing $\left(P_{m}^{*}, \mu_{1}^{*}\right)$ can be derived in terms of closed-form expression via solving (15) and (13) to maximize the sum utilities of both follower and leader games, respectively. This can guarantee Definition 11. In order to implement the Stackelberg equilibrium, the IoT devices first announce a transmit power required to transmit their own information to the SAP with the assistance of the IRSs, which aims to maximize the sum utility of the follower game in (14). For the given power allocation, the PAP coordinate an interference pricing for the payment from each IoT device to maximize the sum utility of the leader game in (12). This procedure can be carried out to maximize their own utility such that both primary and secondary networks come to an agreement on the power allocation at the IoT devices and the interference price. This can confirm the existence of Stackelberg equilibrium. The deviation from this equilibrium will cause loss of performance in terms of sum utility.

\section{Simulation Results}

In this section, simulation results are presented to validate the performance of the proposed scheme. A 2-D coordinate is considered to describe the location of the system model as shown in Fig. 1], where the SAP and PAP are located at $(50,0)$ and $(-50,0)$. Also, the coordinate of all IoT devices as well as all IRSs are denoted as $\left(X_{u, m}, Y_{u, m}\right)$ and $\left(X_{I R S, m}, Y_{I R S, m}\right)$, respectively. It is assumed that the IoT devices and the IRS are randomly deployed within an area centered at $(0,0)$ and $(20,0)$. Also, we assume that each IRS has a square shape with $\alpha=\beta=10 \lambda$, where $\lambda$ denotes the wavelength. In addition, we assume that the number of IoT devices and IRSs is set to be $M=5$, and the spreading gain is assume to be $S=128$. Unless specified, other parameters are: carrier frequency $f=2 \mathrm{GHz}$, gain of transmit antenna $G_{T}=5 \mathrm{~dB}$, gain of receive antenna $G_{R}=5 \mathrm{~dB}$, noise power $\sigma^{2}=-90 \mathrm{dBm}$, interference power $I_{t h}=10^{-5}$. The scheme with IRSs are considered as the benchmark scheme for comparison to highlight the performance of the proposed schemes in terms of sum utility as well as sum rate.

First, we evaluate the sum utility performance of the follower game versus the number of IoT devices $(M)$. The sun utility can be obtained by solving the leader problem (15) for the schemes with/without SIC. In Fig. 4 it can be observed that the sum utility of the follower game increases with $M$ for each scheme. Also, the proposed schemes with/without SIC outperform their counterpart without IRSs, which attributes to the benefits of the IRSs. In addition, the SIC technique plays a positive role in improving the performance of the sum utility. Fig. 5 shows the sum utility of the follower game versus the size of each IRS $\alpha$ or $\beta$, which is from $5 \lambda$ to $40 \lambda$. It can be seen from this figure that the sum utility of the proposed schemes increases with the size of each IRS, and is higher than that without IRSs which remain constant with size of the IRS. This is due to the fact that larger size of IRS can enhance the information reflection efficiency to improve the performance. Similar to Fig. 4, the SIC can further enhance the performance of sum utility for each scheme.

Next, we evaluate the sum utility performance of the leader game, which can be obtained via solving problem (13). Fig. 6 shows that the sum utility of the leader game versus the interference power $I_{t h}$. As can be observed from this figure, the sum utility remains very low at low interference power region and start to rise as the interference power increases for each scheme. In addition, the scheme without SIC provides a larger sum utility than that with SIC at large interference power regime, this is due to the fact that the SIC plays a positive role in improving power efficiency and the sum rate. In addition, the scheme with IRSs obtains a larger sum utility than that with IRSs at large interference power regime, since the IRSs enable a self-sustainable transmission to reduce the transmit power consumption.

Then, the sum rate is evaluated for the schemes without/with SIC, shown in Fig. 7 and Fig. 8, respectively. Fig. 7 demonstrates a three-dimensional plot to show the sum rate performance of the scheme without SIC versus the the number of IoT devices $(M)$ and the size of each IRS $\alpha$ or $\beta$. From this figure, one can observe that the proposed scheme has an increasing trend with number of IoT devices and size of each IRS, respectively, and outperforms that without IRSs which produces an slightly increasing trend with number of IoT 


$$
\mu_{m}^{*}=\frac{\lambda_{m} S\left[\sqrt{L_{\mathrm{IRS}, m}}+\sqrt{L_{d, m}}\right]^{2}}{L_{p, m} \sum_{n>m} P_{n}\left[\sqrt{L_{\mathrm{IRS}, n}}+L_{p, m} \sqrt{L_{d, n}}\right]^{2}+L_{p, m} \sigma^{2}+S L_{p, m} P_{m}\left[\sqrt{L_{\mathrm{IRS}, m}}+\sqrt{L_{d, m}}\right]^{2}} .
$$

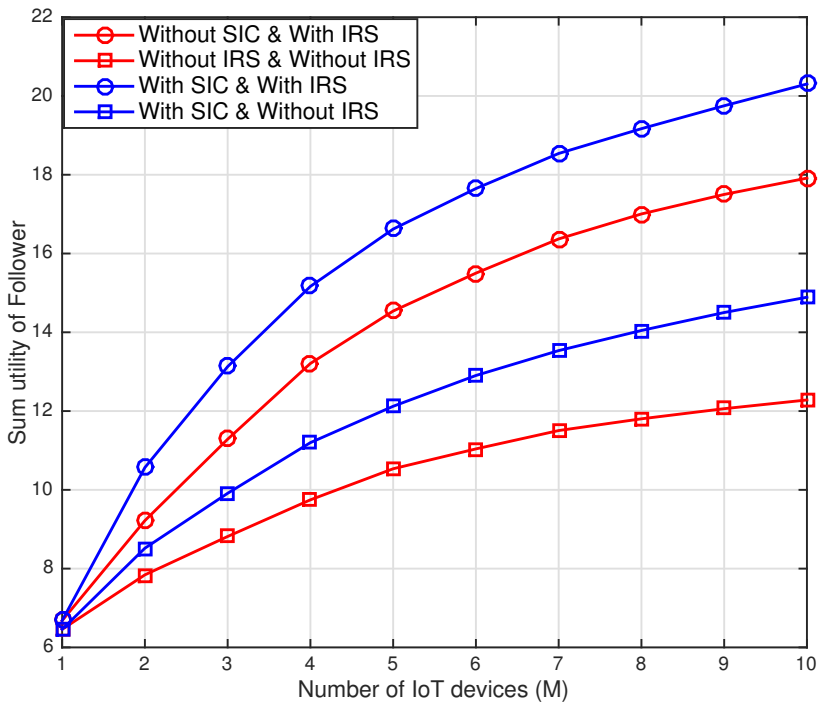

Fig. 4: Sum utility of follower versus number of IoT device $(M)$.

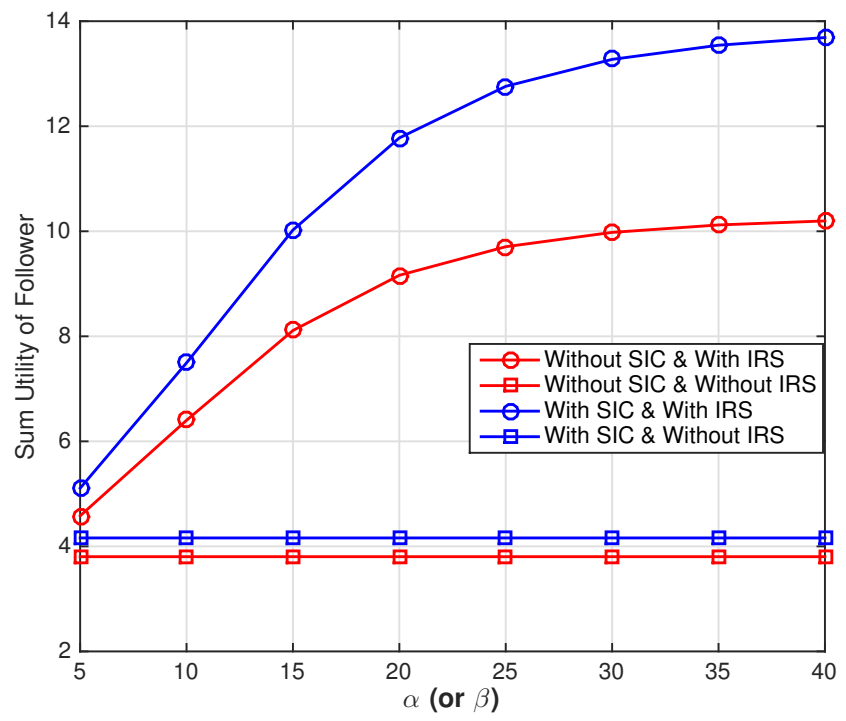

Fig. 5: Sum utility of follower versus size of IRS ( $\alpha$ or $\beta$ ).

devices and remains constant with size of each IRS. Also, the gap between both schemes becomes larger when increasing the number of IoT devices and size of each IRS. Similar trends and arguments can be observed in Fig. 8, i.e., the scheme with SIC provides a better performance than that without SIC in terms of sum rate.

\section{CONCLUSION}

This paper has investigated dedicated IRSs enabled cognitive IoT system. Due to the interference pricing introduced

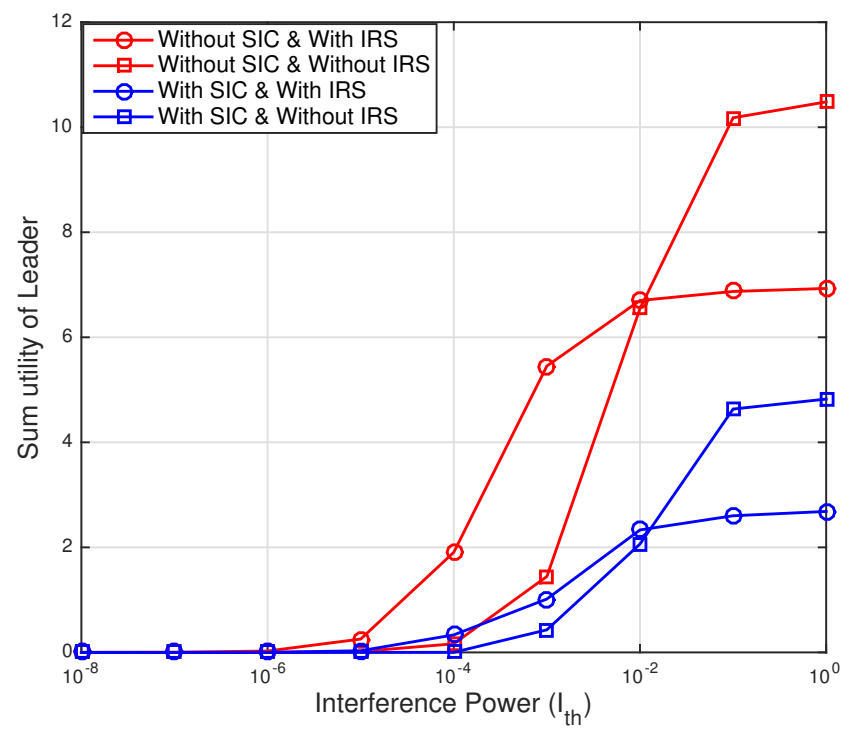

Fig. 6: Sum utility of leader versus interference power $\left(I_{t h}\right)$.

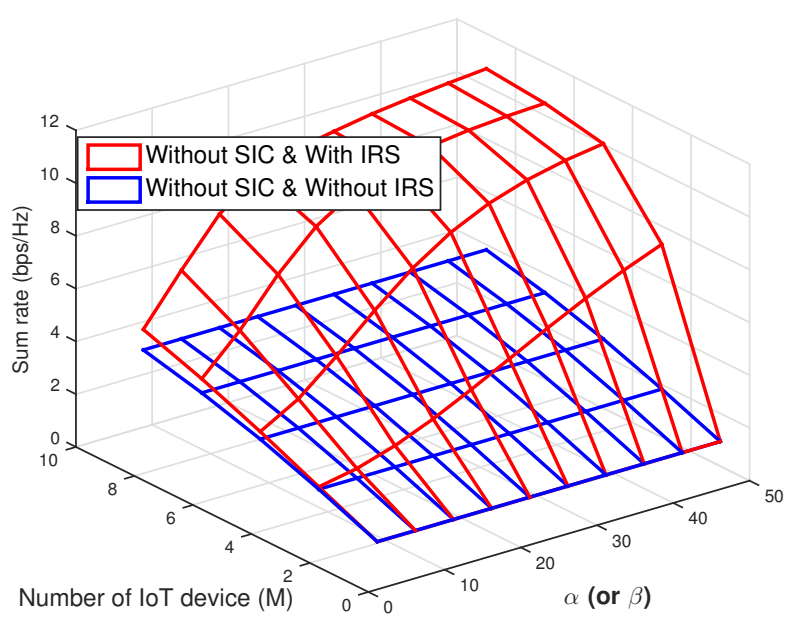

Fig. 7: Three-dimension plot of sum rate in the scheme without SIC.

from the IoT devices, the Stackelberg game has been considered to characterize the interaction between the primary and cognitive networks. Specifically, the leader game is defined as the sum utility function at the PAP subject to the constraints of the interference power and the SINR balancing, while the follower game is modelled as the difference between the sum income from the achievable rate achieved at the SAP and the sum cost of the interference introduced to the PAP. By exploiting the Stackelberg game, we have maximized the sum utility of leader and follower games for their own interest without/with SIC. The Stackelberg equilibrium has been con- 


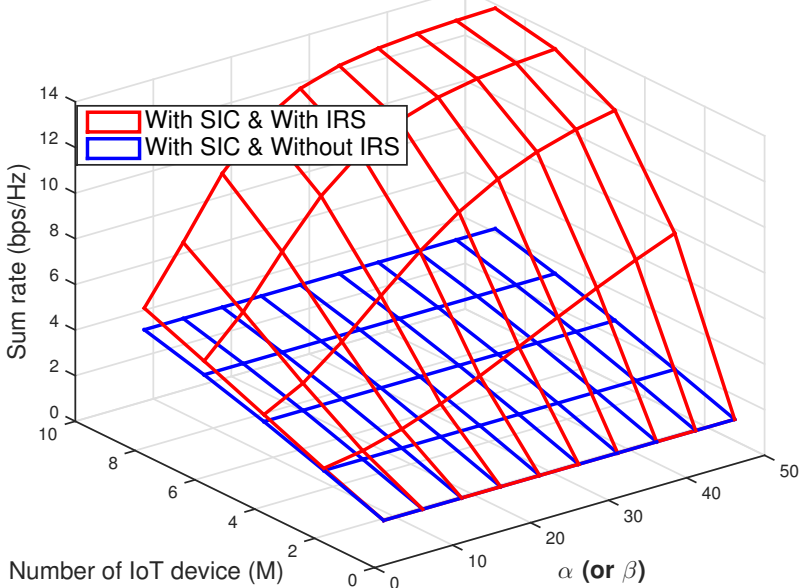

Fig. 8: Three-dimension plot of sum rate in the scheme with SIC.

sidered to optimally derive power allocation and interference pricing of each IoT device in closed-form expressions. Finally, numerical results have been demonstrated the superiority of the proposed scheme over the benchmark scheme.

\section{APPENDIX}

\section{A. Proof of Lemma 1}

In order to proof Lemma 1, we set the first derivative of (14) to zero, which is given by (39) on the top of next page. By exploiting a few of mathematical manipulations, we have

$$
\begin{gathered}
\frac{\sum_{n \neq m} P_{n}\left[\sqrt{L_{\mathrm{IRS}, n}}+\sqrt{L_{d, n}}\right]^{2}}{S}+P_{m}\left[\sqrt{L_{\mathrm{IRS}, m}}+\sqrt{L_{d, m}}\right]^{2} \\
=\frac{\lambda_{m}\left[\sqrt{L_{\mathrm{IRS}, m}}+\sqrt{L_{d, m}}\right]^{2}}{\mu_{m} L_{p, m}}-\frac{\sigma^{2}}{S} .
\end{gathered}
$$

Thus, we can derive (16) by representing (40) in terms of matrix form.

\section{B. Proof of Lemma 2}

By exploiting the SINR balancing constraint (18a), we have the following derivations:

$$
\begin{gathered}
\frac{S P_{m}\left[\sqrt{L_{\mathrm{IRS}, m}}+\sqrt{L_{d, m}}\right]^{2}}{\sum_{n \neq m} P_{n}\left[\sqrt{L_{\mathrm{IRS}, n}}+\sqrt{L_{d, n}}\right]^{2}+\sigma^{2}} \\
=\frac{S P_{n}\left[\sqrt{L_{\mathrm{IRS}, n}}+\sqrt{L_{d, n}}\right]^{2}}{\sum_{j \neq n} P_{j}\left[\sqrt{L_{\mathrm{IRS}, j}}+\sqrt{L_{d, j}}\right]^{2}+\sigma^{2}} \\
\Rightarrow S P_{m}\left[\sqrt{L_{\mathrm{IRS}, m}}+\sqrt{L_{d, m}}\right]^{2} * \\
\left(\sum_{j \neq n} P_{j}\left[\sqrt{L_{\mathrm{IRS}, j}}+\sqrt{L_{d, j}}\right]^{2}+\sigma^{2}\right) \\
-S P_{n}\left[\sqrt{L_{\mathrm{IRS}, n}}+\sqrt{L_{d, n}}\right]^{2} * \\
\left(\sum_{i \neq m} P_{i}\left[\sqrt{L_{\mathrm{IRS}, i}}+\sqrt{L_{d, i}}\right]^{2}+\sigma^{2}\right)=0 .
\end{gathered}
$$

With some mathematical manipulations, we have

$$
\begin{aligned}
& S P_{m} {\left[\sqrt{L_{\mathrm{IRS}, m}}+\sqrt{L_{d, m}}\right]^{2} * } \\
&\left(\sum_{j=1}^{M} P_{j}\left[\sqrt{L_{\mathrm{IRS}, j}}+\sqrt{L_{d, j}}\right]^{2}+\sigma^{2}\right) \\
&-S P_{n}\left[\sqrt{L_{\mathrm{IRS}, n}}+\sqrt{L_{d, n}}\right]^{2} * \\
&\left(\sum_{i=1}^{M} P_{i}\left[\sqrt{L_{\mathrm{IRS}, i}}+\sqrt{L_{d, i}}\right]^{2}+\sigma^{2}\right)=0 . \\
& \Rightarrow S\left(\sum_{j=1}^{M} P_{j}\left[\sqrt{L_{\mathrm{IRS}, j}}+\sqrt{L_{d, j}}\right]^{2}+\sigma^{2}\right) * \\
&\left(P_{m}\left[\sqrt{L_{\mathrm{IRS}, m}}+\sqrt{L_{d, m}}\right]^{2}-P_{n}\left[\sqrt{L_{\mathrm{IRS}, n}}+\sqrt{L_{d, n}}\right]^{2}\right)=0 .
\end{aligned}
$$

From (42), we conclude Lemma 2 due to $S\left(\sum_{j=1}^{M} P_{j}\left[\sqrt{L_{\mathrm{IRS}, j}}+\sqrt{L_{d, j}}\right]^{2}+\sigma^{2}\right)>0$, which completes the proof.

\section{REFERENCES}

[1] A. Yadav and O. A. Dobre, "All technologies work together for good: A glance at future mobile networks," IEEE Wireless Commun., vol. 25, no. 4, pp. 10-16, 2018.

[2] Z. Chu, F. Zhou, Z. Zhu, R. Q. Hu, and P. Xiao, "Wireless powered sensor networks for internet of things: Maximum throughput and optimal power allocation," IEEE Internet Things J., vol. 5, no. 1, pp. 310-321, Feb. 2018.

[3] L. Zhang, Y. Liang, and M. Xiao, "Spectrum sharing for internet of things: A survey," IEEE Wireless Commun., vol. 26, no. 3, pp. 132$139,2019$.

[4] Z. Gao, L. Dai, D. Mi, Z. Wang, M. A. Imran, and M. Z. Shakir, "MmWave massive-MIMO-based wireless backhaul for the $5 \mathrm{G}$ ultradense network," IEEE Wireless Commun., vol. 22, no. 5, pp. 13-21, Oct. 2015

[5] X. Chen, C. Zhong, C. Yuen, and H. Chen, "Multi-antenna relay aided wireless physical layer security," IEEE Commun. Mag., vol. 53, no. 12, pp. 40-46, Dec. 2015.

[6] S. Chen, F. Qin, B. Hu, X. Li, and Z. Chen, "User-centric ultra-dense networks for 5G: challenges, methodologies, and directions," IEEE Wireless Commun., vol. 23, no. 2, pp. 78-85, Apr. 2016.

[7] Renzo, Marco Di, et. al., "Smart radio environments empowered by reconfigurable AI meta-surfaces: an idea whose time has come," EURASIP J. Wirel. Commun. Netw., no. 129, pp. 1-20, May 2019.

[8] S. Dang, O. Amin, B. Shihada, and M. Alouini, "What should 6G be?," Nat. Electron., vol. 3, no. 1, pp. 20-29, Jan. 2020.

[9] Q. Wu and R. Zhang, "Intelligent reflecting surface enhanced wireless network via joint active and passive beamforming," IEEE Trans Wireless Commun., vol. 18, no. 11, pp. 5394-5409, Nov. 2019.

[10] Q. Wu and R. Zhang, "Towards smart and reconfigurable environment: Intelligent reflecting surface aided wireless network," IEEE Commun. Mag., vol. 58, no. 1, pp. 106-112, Jan. 2020.

[11] Z. Chu, W. Hao, P. Xiao, and J. Shi, "Intelligent reflecting surface aided multi-antenna secure transmission," IEEE Wireless Commun. Lett., vol. 9, no. 1, pp. 108-112, Jan. 2020.

[12] H. Shen, W. Xu, S. Gong, Z. He, and C. Zhao, "Secrecy rate maximization for intelligent reflecting surface assisted multi-antenna communications," IEEE Commun. Lett., vol. 23, no. 9, pp. 1488-1492, Sept. 2019.

[13] C. Pan, H. Ren, K. Wang, W. Xu, M. Elkashlan, A. Nallanathan, and L. Hanzo, "Multicell MIMO communications relying on intelligent reflecting surfaces," IEEE Trans. Wireless Commun., vol. 19, no. 8, pp. 5218-5233, Aug. 2020

[14] Q. Wu and R. Zhang, "Weighted sum power maximization for intelligent reflecting surface aided SWIPT," IEEE Wireless Commun. Lett., vol. 9, no. 5, pp. 586-590, May 2020. 


$$
\frac{\partial U_{F}}{\partial P_{m}}=\frac{\lambda_{m} S\left[\sqrt{L_{\mathrm{IRS}, m}}+\sqrt{L_{d, m}}\right]^{2}}{\sum_{n \neq m} P_{n}\left[\sqrt{L_{\mathrm{IRS}, n}}+\sqrt{L_{d, n}}\right]^{2}+\sigma^{2}+S P_{m}\left[\sqrt{L_{\mathrm{IRS}, m}}+\sqrt{L_{d, m}}\right]^{2}}=\mu_{m} L_{p, m} .
$$

[15] Q. Wu and R. Zhang, "Joint active and passive beamforming optimization for intelligent reflecting surface assisted SWIPT under QoS constraints," IEEE J. Sel. Areas Commun., vol. 38, no. 8, pp. 17351748, Aug. 2020.

[16] C. Pan, H. Ren, K. Wang, M. Elkashlan, A. Nallanathan, J. Wang, and L. Hanzo, "Intelligent reflecting surface aided MIMO broadcasting for simultaneous wireless information and power transfer," IEEE J. Sel. Areas Commun., vol. 38, no. 8, pp. 1719-1734, Aug. 2020.

[17] S. Zhang and R. Zhang, "Intelligent reflecting surface aided multiple access: Capacity region and deployment strategy," in IEEE SPAWC, Atlanta, GA, USA, pp. 1-5, 2020.

[18] Y. Zheng, S. Bi, Y. J. Zhang, Z. Quan, and H. Wang, "Intelligent reflecting surface enhanced user cooperation in wireless powered communication networks," IEEE Wireless Commun. Lett., 2020.

[19] T. Bai, C. Pan, Y. Deng, M. Elkashlan, A. Nallanathan, and L. Hanzo, "Latency minimization for intelligent reflecting surface aided mobile edge computing," IEEE J. Sel. Areas Commun., vol. 38, no. 11, pp. 2666-2682, Nov. 2020.

[20] T. Bai, C. Pan, H. Ren, Y. Deng, M. Elkashlan, and A. Nallanathan, "Resource allocation for intelligent reflecting surface aided wireless powered mobile edge computing in OFDM systems," https://arxiv.org/ abs/2003.05511. 2020.

[21] O. Ozdogan, E. Bjornson, and E. G. Larsson, "Intelligent reflecting surfaces: Physics, propagation, and pathloss modeling," IEEE Wireless Commun. Lett., pp. 1-1, Dec. 2019.

[22] G. Geraci, M. Egan, J. Yuan, A. Razi, and I. B. Collings, "Secrecy sumrates for multi-user MIMO regularized channel inversion precoding," IEEE Trans. Commun., vol. 60, no. 11, pp. 3472-3482, Nov. 2012.

[23] A. Mukherjee and A. L. Swindlehurst, "Detecting passive eavesdroppers in the MIMO wiretap channel," in IEEE ICASSP, Tokyo, Japan, Mar. 2012.

[24] H. Yu, L. Gao, Z. Li, X. Wang, and E. Hossain, "Pricing for uplink power control in cognitive radio networks," IEEE Trans. Veh. Technol., vol. 59, no. 4, pp. 1769-1778, 2010. 